\title{
Thermal Conductivity of Ordered Mesoporous Titania Films Made from Nanocrystalline Building Blocks and Sol-Gel Reagents
}

\author{
Thomas Coquil, ${ }^{\dagger}$ Christian Reitz, ${ }^{\ddagger}$ Torsten Brezesinski, ${ }^{*, \ddagger}$ E. Joseph Nemanick, ${ }^{\S}$ \\ Sarah H. Tolbert, $*$, and Laurent Pilon $*, \dagger$ \\ Henry Samueli School of Engineering and Applied Science, Mechanical and Aerospace Engineering \\ Department, 420 Westwood Plaza, University of California, Los Angeles, California 90095, Institute of \\ Physical Chemistry, Justus-Liebig-University Giessen, Heinrich-Buff Ring 58, Giessen 35392, and Department \\ of Chemistry and Biochemistry, 607 Charles E. Young Drive East, Box 951569, University of California, \\ Los Angeles, California 90095-1569
}

Received: April 12, 2010; Revised Manuscript Received: June 10, 2010

\begin{abstract}
This paper reports the cross-plane thermal conductivity of amorphous and crystalline mesoporous titania thin films synthesized by evaporation-induced self-assembly. Both sol-gel and nanocrystal-based mesoporous films were investigated, with average porosities of $30 \%$ and $35 \%$, respectively. The pore diameter ranged from 7 to $30 \mathrm{~nm}$ and film thickness from 60 to $370 \mathrm{~nm}$, while the average wall thickness varied from 3 to 50 $\mathrm{nm}$. The crystalline domain sizes in sol-gel films varied from 12 to $13 \mathrm{~nm}$, while the nanocrystal-based films consisted of monodisperse nanocrystals $9 \mathrm{~nm}$ in diameter. The cross-plane thermal conductivity was measured at room temperature using the $3 \omega$ method. The average thermal conductivity of the amorphous sol-gel mesoporous titania films was $0.37 \pm 0.05 \mathrm{~W} / \mathrm{m} \cdot \mathrm{K}$. It did not show strong dependence on pore diameter, wall thickness, and film thickness for sol-gel amorphous mesoporous titania thin films. This result can be attributed to the fact that heat is carried, in the amorphous matrix, by localized nonpropagating vibrational modes. The thermal conductivity of crystalline sol-gel mesoporous titania thin films was significantly larger at $1.06 \pm$ $0.04 \mathrm{~W} / \mathrm{m} \cdot \mathrm{K}$ and depended on the organic template used to make the films. The thermal conductivity of nanocrystal-based thin films was $0.48 \pm 0.05 \mathrm{~W} / \mathrm{m} \cdot \mathrm{K}$ and significantly lower than that of the crystalline sol-gel mesoporous thin films. This was due to the fact that the nanocrystals were not as well interconnected as the crystalline domains in the crystalline sol-gel films. These results suggest that both connectivity and size of the nanocrystals or the crystalline domains can provide control over thermal conductivity in addition to porosity.
\end{abstract}

\section{Introduction}

Mesoporous titania $\left(\mathrm{TiO}_{2}\right)$ thin films have received significant attention due to their wide range of applications. They have been used in dye-sensitized solar cells for their wide band gap semiconductor properties. ${ }^{1}$ They have also been considered for solid oxide fuel cells as high proton conductivity porous exchange membranes. ${ }^{2}$ Mesoporous $\mathrm{TiO}_{2}$ is also a very interesting material for controlled delivery of chemicals, highly specific chemical sensors, and membranes ${ }^{3}$ thanks to its tunable pore size and structure. ${ }^{4}$ In addition, the high oxidative potential of titania gives it strong photocatalytic activity for water and air purification applications. ${ }^{5,6}$ Moreover, mesoporous $\mathrm{TiO}_{2}$ films have been used for optical coatings, emissive displays, or optoelectronics $^{7}$ due to their large surface area and controlled nanoscale morphologies coupled with relatively high refractive index $(\geq 1.6)$. They are also of interest for electrical energy storage applications such as electrochemical capacitors. ${ }^{8-10}$

* To whom correspondence should be addressed. E-mail: torsten.brezesinski@phys.chemie.unigiessen.de (T.B.); phone +1 (310)-2064767, fax +1 (310)-206-4038, e-mail tolbert@ chem.ucla.edu (S.H.T); phone +1 (310)-206-5598, fax +1 (310)-206-2302, e-mail pilon@seas.ucla.edu (L.P.).

Mechanical and Aerospace Engineering Department, University of California, Los Angeles.

$¥$ Justus-Liebig-University of Giessen.

$\S$ Department of Chemistry and Biochemistry, University of California, Los Angeles.
Finally, Choi et al. ${ }^{11}$ have also identified mesoporous titania thin films as promising thermal insulating materials for infrared sensors.

Knowledge of the thermal properties of such films is necessary for their practical implementation in devices which typically operate at room temperature. A few studies have also reported the thermal conductivity of dense (nonporous) amorphous and crystalline titania films. ${ }^{12-15}$ Note that titania does not naturally have a bulk amorphous phase, but the theoretical minimum thermal conductivity of bulk amorphous sputtered titania at room temperature was estimated by Cahill and Hallen ${ }^{12}$ to be $1.5 \mathrm{~W} / \mathrm{m} \cdot \mathrm{K}$. Choi et al. ${ }^{11}$ reported the only thermal conductivity measurement for a single mesoporous $\mathrm{TiO}_{2}$ thin film. The authors considered a $250 \mathrm{~nm}$ thick amorphous templated film at room temperature. Unfortunately, the authors did not report the film porosity and morphology.

The goal of the present study is to investigate the effect of (i) porosity and pore spatial arrangement and (ii) crystallinity of the $\mathrm{TiO}_{2}$ matrix on the thermal conductivity of the templated films at room temperature. First, both synthesis and characterization of the mesoporous titania films are described. Then, the cross-plane thermal conductivity of all samples is reported and discussed.

\section{Method and Experiments}

2.1. Sample Film Preparation. In this study, both nontemplated (i.e., mostly nonporous) and templated thin films with 
amorphous and crystalline frameworks were synthesized. The synthesis was based on calcination of periodic polymer/titania composites produced by evaporation-induced self-assembly. Two different types of organic templates were used, namely, poly(ethylene-co-butylene) ${ }_{89}$-block-poly(ethylene oxide) ${ }_{79}$, also referred to as KLE, ${ }^{8}$ and poly(ethylene oxide) ${ }_{20}$-block-poly(propylene oxide $)_{70}$-block-poly(ethylene oxide ${ }_{20}$ triblock copolymer $\left(\mathrm{EO}_{20} \mathrm{PO}_{70} \mathrm{EO}_{20}\right)$, also referred to as $\mathrm{P} 123$. Their use resulted in films with different pore sizes and interpore spacings. In addition, two types of mesoporous titania thin films were synthesized, namely, sol-gel-derived films using both KLE and P123 and nanocrystal-based films synthesized using KLE. Synthesis of both the sol-gel and nanocrystal-based mesoporous titania thin films using KLE followed methods reported by Fattakhova-Rohlfing et al. ${ }^{8}$ and Brezesinski et al., ${ }^{9}$ respectively. Synthesis of the sol-gel cubic mesoporous titania thin films using P123 followed a method reported by Alberius et al. ${ }^{16}$

Synthesis of the sol-gel cubic mesoporous titania framework using KLE was accomplished using a mixture of KLE, ethanol $(\mathrm{EtOH})$, water $\left(\mathrm{H}_{2} \mathrm{O}\right)$, and titanium tetrachloride $(99.9 \%)\left(\mathrm{TiCl}_{4}\right)$. First, $600 \mathrm{mg}$ of $\mathrm{TiCl}_{4}$ was carefully combined with $3 \mathrm{~mL}$ of EtOH. After $10 \mathrm{~min}, 100 \mathrm{mg}$ of KLE dissolved in $3 \mathrm{~mL}$ of EtOH and $0.5 \mathrm{~mL}$ of double-distilled $\mathrm{H}_{2} \mathrm{O}$ were added. Thin films were produced via dip-coating on silicon substrates at a $20 \%$ relative humidity $(\mathrm{RH})$ and a constant withdrawal rate between 1 and $10 \mathrm{~mm} / \mathrm{s}$. Films were then aged at $300{ }^{\circ} \mathrm{C}$ for $12 \mathrm{~h}$ prior to template removal to prevent loss of mesoscale order during thermal treatment. Amorphous films were obtained by calcination using a $5{ }^{\circ} \mathrm{C} / \mathrm{min}$ ramp to $400{ }^{\circ} \mathrm{C}$ followed by a 10 min soak time. Alternatively, crystalline films were obtained by calcination using a $1 \mathrm{~h}$ heating ramp from room temperature to $600{ }^{\circ} \mathrm{C}$ with a 10 min hold. Brezesinski et al. ${ }^{9}$ and FattakhovaRohlfing et al. ${ }^{8}$ have shown, using wide-angle $\mathrm{X}$-ray diffraction, that films obtained this way can be considered as fully crystalline and exclusively anatase phase. ${ }^{8}$

Synthesis of the sol-gel cubic mesoporous titania framework using P123 was accomplished using a mixture of P123, EtOH, 12.1 M hydrochloric acid $(\mathrm{HCl})$, and tetraethyl orthotitanate (TEOT) in the mass ratio P123:EtOH:HCl:TEOT $=1: 15: 3.2$ : 4.2. A polymer solution was made by dissolving $1 \mathrm{~g}$ of P123 in $14 \mathrm{~g}$ of EtOH. A titania precursor solution was made by mixing $3.2 \mathrm{~g}$ of $\mathrm{HCl}$ with $4.2 \mathrm{~g}$ of TEOT under nitrogen atmosphere. This solution was stirred for $10 \mathrm{~min}$ until it became homogeneous and clear. The polymer solution was then added followed by a $20 \mathrm{~min}$ stirring at room temperature. Films were dip-coated from the solution onto silicon substrates at a withdrawal rate of $1-6 \mathrm{~cm} / \mathrm{min}$ and a $\mathrm{RH}$ of $20 \%$. After deposition, films were aged overnight at $-20{ }^{\circ} \mathrm{C}$, followed by 1 day at $60^{\circ} \mathrm{C}$. Amorphous films were obtained by heating the films, in air, up to $300{ }^{\circ} \mathrm{C}$ using a $1{ }^{\circ} \mathrm{C} / \mathrm{min}$ ramp followed by $6 \mathrm{~h}$ of soak time. Alternatively, crystalline anatase films were obtained by heating the samples, in air, up to $550{ }^{\circ} \mathrm{C}$ using a $1.5^{\circ} \mathrm{C} / \mathrm{min}$ ramp followed by $6 \mathrm{~h}$ of soak time.

Synthesis of the nanocrystal-based titania framework using KLE was accomplished by first preparing an anatase nanoparticle solution. ${ }^{10} \mathrm{In}$ a water-free container, $0.5 \mathrm{~mL}$ of $\mathrm{TiCl}_{4}$ was slowly added to $2 \mathrm{~mL}$ of EtOH and then combined with $10 \mathrm{~mL}$ of anhydrous benzyl alcohol. The container was loosely sealed and the solution heated at $80{ }^{\circ} \mathrm{C}$ for $9 \mathrm{~h}$. To isolate the nanocrystalline particles, $1 \mathrm{~mL}$ of the suspension was precipitated in $12 \mathrm{~mL}$ of diethyl ether and centrifuged at $5000 \mathrm{rpm}$. The resulting white $\mathrm{TiO}_{2}$ powder was then dispersed in $3 \mathrm{~mL}$ of $\mathrm{EtOH}$ and sonicated for $2 \mathrm{~h}$, yielding a slightly opaque solution. Then, $60 \mathrm{mg}$ of KLE dissolved in $0.5 \mathrm{~mL}$ of EtOH
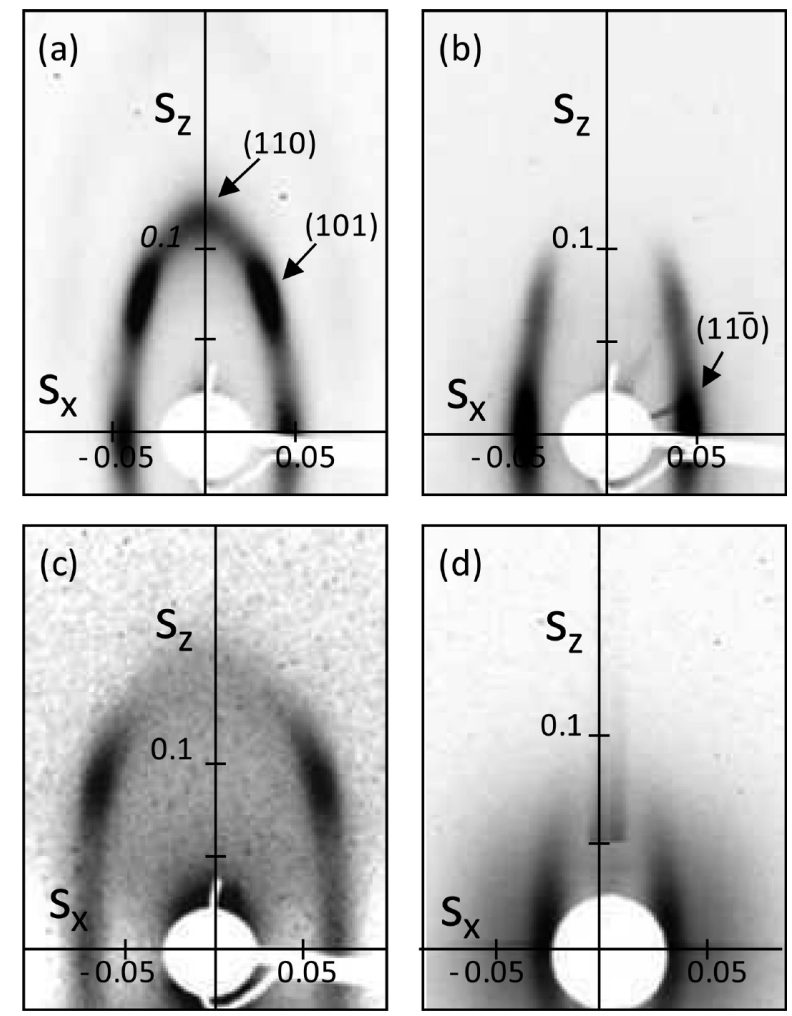

Figure 1. 2D-SAXS patterns obtained on sol-gel type KLE-templated films heated to (a) $400{ }^{\circ} \mathrm{C}$ (amorphous matrix) and (b) $600{ }^{\circ} \mathrm{C}$ (crystalline matrix), (c) sol-gel type P123-templated films heated to $300{ }^{\circ} \mathrm{C}$, and (d) nanocrystal-based KLE-templated films heated to 600 ${ }^{\circ} \mathrm{C}$. Scattering vector $\vec{S}$ components are given in $\mathrm{nm}^{-1} ; \mid \vec{S} \|=(2 / \lambda) \sin \theta$.

was added to $4 \mathrm{~mL}$ of this anatase nanoparticle solution (content: $15 \mathrm{mg} / \mathrm{mL}$ ). Once the solution was homogeneous, $0.2 \mathrm{~mL}$ of double-distilled water was added. Thin films were dip-coated from the solution onto silicon substrates at $30 \% \mathrm{RH}$. Calcination was performed using a $2 \mathrm{~h}$ ramp from room temperature to 600 ${ }^{\circ} \mathrm{C}$, followed by a $1 \mathrm{~h}$ soak.

Finally, nontemplated (i.e., mostly nonporous) amorphous and crystalline $\mathrm{TiO}_{2}$ films were prepared by a procedure similar to that of the mesoporous sol-gel films but without the use of polymer template. These films were used to validate the thermal conductivity measurements.

2.2. Film Characterization. Films characterization was performed using both one- and two-dimensional small-angle X-ray scattering (1D-SAXS and 2D-SAXS), scanning and transmission electron microscopy (SEM and TEM), and wideangle X-ray diffraction (WAXD) measurements. 1D-SAXS measurements were performed for every sample with a Panalytical X'Pert PRO MPD diffractometer utilizing a mirror-mirror $(\theta-2 \theta)$ geometry. SEM micrographs and bright field TEM micrographs were obtained using a JEOL 6700F instrument, and a Philips CM30-ST microscope, respectively. 2D-SAXS patterns were collected both on beamline 1-4 at the Stanford Synchrotron Radiation Laboratory and on a Nonius rotating anode with MarCCD area detector. WAXD measurements were carried out on a D8-GADDS diffractometer from Bruker instruments ( $\mathrm{Cu} \mathrm{K}-\alpha$ radiation) as well as on the Panalytical X'Pert PRO MPD diffractometer.

Mesoporous Sol-Gel-Based Thin Films. Figure 1 shows typical 2D-SAXS patterns for (a) KLE-templated amorphous, (b) KLE-templated crystalline, and (c) P123-templated amorphous sol-gel mesoporous titania thin films as well as for (d) 


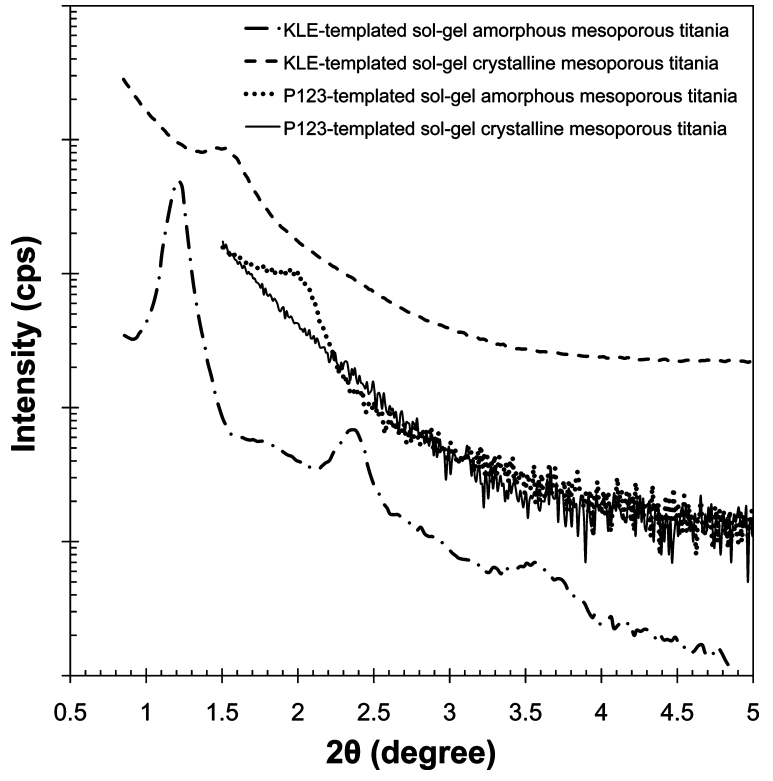

Figure 2. 1D-SAXS measurements for amorphous and crystalline sol-gel mesoporous $\mathrm{TiO}_{2}$ thin films synthesized using KLE and P123.

nanocrystal-based KLE-templated samples. Data were collected at an angle of incidence $\beta=5^{\circ}$. Figures $1 \mathrm{a}$ and $\mathrm{b}$ show the evolution of the body-centered cubic (bcc) architecture with (110) orientation of the KLE-templated sol-gel $\mathrm{TiO}_{2}$ films upon thermal treatment. Figure 1c illustrates the face-centered cubic (fcc) architecture with (111) orientation of the P123-derived amorphous $\mathrm{TiO}_{2}$ films. While the 2D-SAXS images are the best way to analyze the symmetry and orientation of the mesoporous structure, integrated 1D patterns are easier for quantitative comparison of the size and repeat distances of the periodic structures.

Figure 2 shows typical 1D-SAXS measurements for the KLEand P123-templated sol-gel mesoporous $\mathrm{TiO}_{2}$ films. The data indicates that the pores of the amorphous thin films were highly ordered and were used to quantify the out-of-plane repeat distance for both nanostructures. Figures $1 \mathrm{~b}$ and 2 also indicate that the nanoscale periodicity of the KLE-templated crystalline mesoporous thin films was retained after thermal treatment at $600{ }^{\circ} \mathrm{C} .{ }^{8,17}$ The slight loss of out-of-plane periodicity of the KLE-derived films was due to both the small number of repeat units in the direction normal to the substrate and the fact that some restructuring of the pore network occurred upon crystallization. On the contrary, the 1D-SAXS of the P123-derived mesoporous titania thin films heated to $550{ }^{\circ} \mathrm{C}$ (Figure 2) show a complete loss of out-of-plane periodicity upon crystallization due to the thinner pore walls. This was accompanied by shrinking in the direction normal to the substrate during thermal treatment.

Figure 3 shows SEM micrographs of (a) KLE-templated amorphous, (b) KLE-templated crystalline, (c) P123-templated amorphous, and (d) P123-templated crystalline sol-gel $\mathrm{TiO}_{2}$ thin films. These micrographs confirm the highly ordered mesoporous structure of the amorphous films. Figure $3 \mathrm{~d}$ also shows the restructuring of the P123-templated crystalline mesoporous thin films upon heating which was not observed for the KLE-templated $\mathrm{TiO}_{2}$ films (Figure 3b). This restructuring was associated with random crystal growth and partial film collapse. The in-plane pore-to-pore distance was estimated from 2D-SAXS patterns except for P123-templated crystalline films which were too disordered to produce quality 2D-SAXS data.
Then, SEM micrographs were used instead. The wall thickness $t_{\text {wall }}$ separating two adjacent pores was also determined from SEM images. The P123- and KLE-templated amorphous mesoporous sol-gel $\mathrm{TiO}_{2}$ thin films consisted of ellipsoidal pores of horizontal diameter $d$ organized in fcc and bcc lattices, respectively (Figures 3a and 3c). They exhibited an in-plane pore diameter $d$ varying from 7 to $12 \mathrm{~nm}$ and 15 to $19 \mathrm{~nm}$, respectively, while the wall thicknesses $t_{\text {wall }}$ ranged from 3 to 6 $\mathrm{nm}$ and from 8 to $12 \mathrm{~nm}$, respectively. The KLE-templated sol-gel films showed similar pore dimensions in crystalline and amorphous states as also observed by Fattakhova-Rohlfing et al. ${ }^{8}$

Figure 4 shows WAXD patterns of (i) the KLE-templated nanocrystal-based mesoporous $\mathrm{TiO}_{2}$ films, (ii) the KLE-templated crystalline sol-gel mesoporous films, (iii) the dense (nontemplated) crystalline sol-gel type films, and (iv) the P123templated sol-gel crystalline mesoporous films. Crystallization of the KLE-templated sol-gel type mesoporous films resulted in $12-13 \mathrm{~nm}$ anatase domains in the $\mathrm{TiO}_{2}$ matrix as indicated by the width of the diffraction peak around $2 \theta=25^{\circ}$. The P123templated sol-gel crystalline mesoporous films exhibited smaller $9 \mathrm{~nm}$ anatase domains. The anatase domains within the KLE sol-gel films were randomly oriented and similar in size to the wall thickness dimensions. ${ }^{9}$

The film porosity was not directly measured in this study. However, Fattakhova-Rohlfing et al. ${ }^{8}$ performed $\mathrm{Kr}$ adsorption measurements on identical samples. Porosity can therefore be assumed to be close to $30 \pm 2 \%$ for the P123 and KLE amorphous mesoporous films as well as for the KLE-templated crystalline mesoporous titania thin films. However, the porosity of the P123-templated crystalline mesoporous titania thin films was shown to be lowered to approximately $13 \% .^{8}$ Those results were in good agreement with the dimensions obtained from SEM/XRD.

Mesoporous KLE Nanocrystal-Based Thin Films. The nanoscale structure of these films has already been extensively described by Brezesinski et al. ${ }^{9}$ Figures $3 \mathrm{e}$ and $3 \mathrm{f}$ show respectively SEM and bright field TEM micrographs of the disordered but macroscopically homogeneous architecture of these films with pores averaging 17- $25 \mathrm{~nm}$ in diameter. Higher magnification SEM micrographs reveal a bimodal structure with 1-4 nm micropores (due to random nanocrystal agglomeration) located between the larger KLE-derived mesopores. ${ }^{9}$ They also show that the pore walls are $15-25 \mathrm{~nm}$ thick and comprised of several layers of nanocrystals. ${ }^{9}$ According to WAXD measurements (Figure 4), the $\mathrm{TiO}_{2}$ nanocrystals are $9 \mathrm{~nm}$ in diameter and correspond to anatase titania. Finally, 2D-SAXS measurements (Figure 1d) were characteristic of a disordered but homogeneous pore system. ${ }^{9}$ Porosity was measured by toluene adsorption and found to be $35 \pm 2 \%$. $^{9}$

Dense Crystalline Sol-Gel Type Thin Films. WAXD measurements in Figure 4 showed that the dense crystalline sol-gel type thin films were made of anatase titania with crystalline domains about $30 \mathrm{~nm}$ in diameter.

Finally, spectral normal reflectance measurements were performed on all films for wavelengths between 400 and 900 $\mathrm{nm}$. The film thickness $t_{\mathrm{f}}$ of all samples was retrieved by inverse method as previously described ${ }^{18}$ and ranged from 60 to 370 $\mathrm{nm}$. Results were confirmed by SEM micrographs.

2.3. Thermal Conductivity Measurements. The cross-plane thermal conductivity of the highly ordered mesoporous $\mathrm{TiO}_{2}$ thin films was measured at room temperature using the $3 \omega$ method. ${ }^{19}$ As discussed by Olson et al., ${ }^{20}$ "the periodic nature of the $3 \omega$ method makes it inherently insensitive to the radiative 

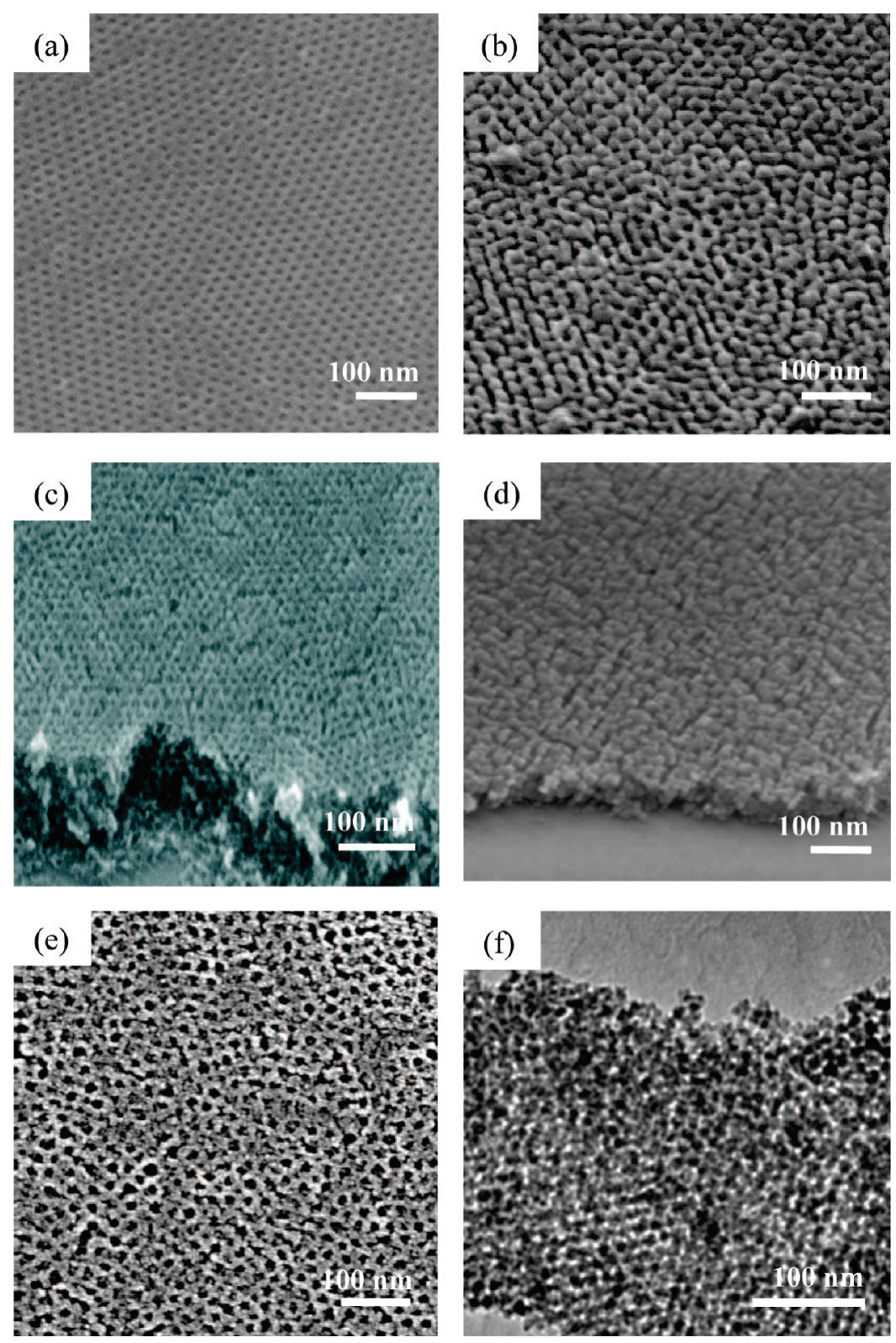

Figure 3. SEM micrographs of (a) KLE amorphous, (b) KLE crystalline, (c) P123 amorphous, and (d) $\mathrm{P} 123$ crystalline sol-gel mesoporous TiO 2 thin films along with SEM (e) and TEM (f) micrographs of nanocrystal-based mesoporous $\mathrm{TiO}_{2}$ thin films.

and convective losses that can adversely affect other techniques." Moreover, samples were covered by a cap located about $5 \mathrm{~mm}$ above the metallic wire to reduce heat losses by natural convection. The associated Rayleigh number was lower than 10 , which was much smaller than the critical value of about 1700 above which convection dominates over conduction for this configuration. ${ }^{21}$ Finally, the sample's thermal conductivity was much larger than that of air. Therefore, heat losses to the surrounding could be safely neglected. Principles, experimental apparatus, experimental procedure, and validation of the method have already been described elsewhere ${ }^{18}$ and need not be repeated. Based on a previous study, ${ }^{18}$ the uncertainty of the thermal conductivity measurements associated with the $3 \omega$ method was estimated to be $\pm 15 \% .^{18}$ The latter accounts for potential errors due to both the sample film preparation and the various steps involved in the $3 \omega$ method, i.e., sample dehydration, variations in PECVD nitride film thickness and inhomogeneity, pattern resolution during photolithography, metal deposition, thickness and width during evaporation and liftoff, and finally bias errors in the $3 \omega$ electrical measurements.
Here, the method was further validated by comparing thermal conductivity measurements for nontemplated amorphous and crystalline sol-gel type titania thin films with data reported in the literature. ${ }^{12,13}$ Although these films do not have regular mesoporosity, they are solution processed and thus have some small fractional porosity. However, their thermal conductivity should be similar to that of purely dense amorphous and crystalline titania films. ${ }^{12,13}$ Results were also compared with predictions by the model developed by Lee and Cahill ${ }^{22}$ and expressed as

$$
k_{\mathrm{f}}=\frac{k_{\mathrm{i}}}{1+k_{\mathrm{i}} r_{\mathrm{c}} / t_{\mathrm{f}}}
$$

where $k_{\mathrm{i}}$ is the intrinsic thermal conductivity of the mesoporous phase which is independent of thickness. The measured thermal conductivity accounts not only for the film thermal resistance but also for the contribution of the interface thermal resistance 


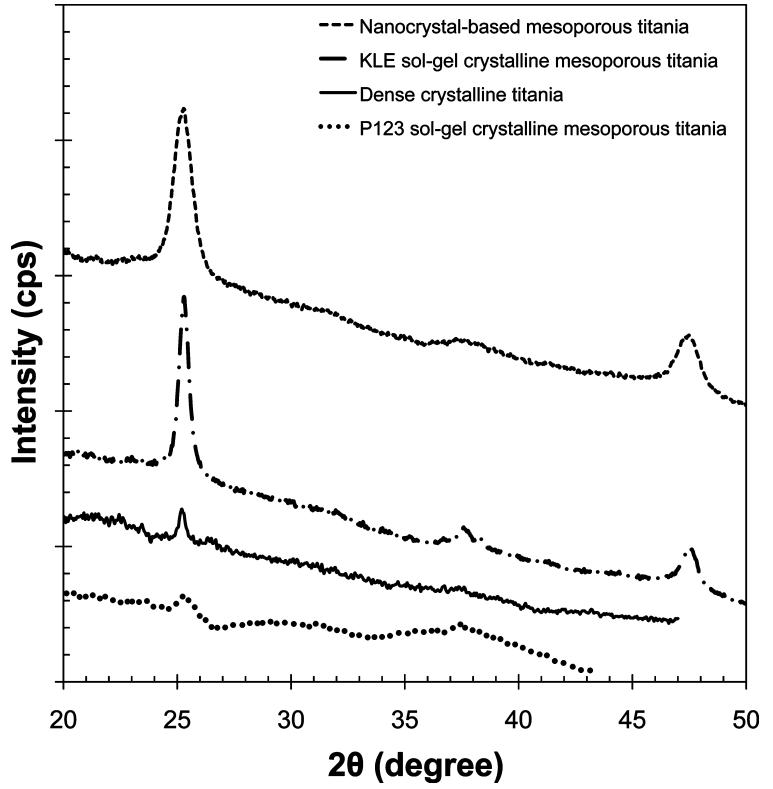

Figure 4. WAXD patterns of nanocrystal-based mesoporous films, KLE- and P123-templated sol-gel mesoporous films, and dense crystalline sol-gel $\mathrm{TiO}_{2}$ films.

$r_{\mathrm{c}}$ due to the interfaces between (i) the film and the silicon substrate and (ii) the metallic heater and the film. The values of $k_{\mathrm{i}}$ used in eq 1 were obtained from the literature as 1.5 $\mathrm{W} / \mathrm{m} \cdot \mathrm{K}$ for amorphous sputtered titania films ${ }^{12,14}$ and 8.4 $\mathrm{W} / \mathrm{m} \cdot \mathrm{K}$ for polycrystalline anatase titania. ${ }^{23}$ Although the nontemplated sol-gel amorphous titania films considered in this study may have slightly lower densities, $k_{\mathrm{i}}=1.5 \mathrm{~W} / \mathrm{m} \cdot \mathrm{K}$ was chosen as their intrinsic thermal conductivity. The value of $r_{\mathrm{c}}$ used in eq 1 was taken as $4 \times 10^{-8} \mathrm{~m}^{2} \cdot \mathrm{K} / \mathrm{W}$ for both amorphous and crystalline $\mathrm{TiO}_{2}$ films as suggested by the values of $2.0-4.0$ $\times 10^{-8} \mathrm{~m}^{2} \cdot \mathrm{K} / \mathrm{W}$ used by Mun et al. ${ }^{13}$ for sputtered titania thin films as well as that previously reported for silicon nitride 22 and silicon dioxide thin films. ${ }^{24}$

Figure 5 compares the thermal conductivity measurements of the nontemplated $\mathrm{TiO}_{2}$ films synthesized in the present study with predictions from eq 1 with $r_{\mathrm{c}}=4 \times 10^{-8} \mathrm{~m}^{2} \cdot \mathrm{K} / \mathrm{W}$ and experimental data reported in refs 12 and 13 . The measured thermal conductivity of amorphous dense films agrees well with previously reported data. The slight deviation from the thermal conductivity model predictions can be attributed to the choice of $k_{\mathrm{i}}$ corresponding to dense sputtered titania thin films ${ }^{12}$ which were denser than sol-gel films used in the present study. ${ }^{25}$ The larger difference observed with previously reported data ${ }^{13}$ for the dense polycrystalline $\mathrm{TiO}_{2}$ films may be due to differences in the size of the crystalline domains. Overall, the experimental setup and the associated analysis give good results and, in turn, can be utilized to measure the thermal conductivity of the synthesized mesoporous $\mathrm{TiO}_{2}$ thin films.

\section{Results and Discussion}

The values of the power dissipated per unit length within the aluminum wire and its thermal coefficient of resistance were measured for each sample and found to be $33 \pm 3 \mathrm{~W} / \mathrm{m} \cdot \mathrm{K}$ and $2.1 \pm 0.3 \times 10^{-3} \mathrm{~K}^{-1}$, respectively. All samples were dehydrated for a minimum of $12 \mathrm{~h}$ at $160{ }^{\circ} \mathrm{C}$ on a hot plate before any measurement. The thermal conductivity of each mesoporous $\mathrm{TiO}_{2}$ film was measured more than eight times using the same heater. The results were then averaged, and the standard deviation was estimated. Table 1 summarizes the

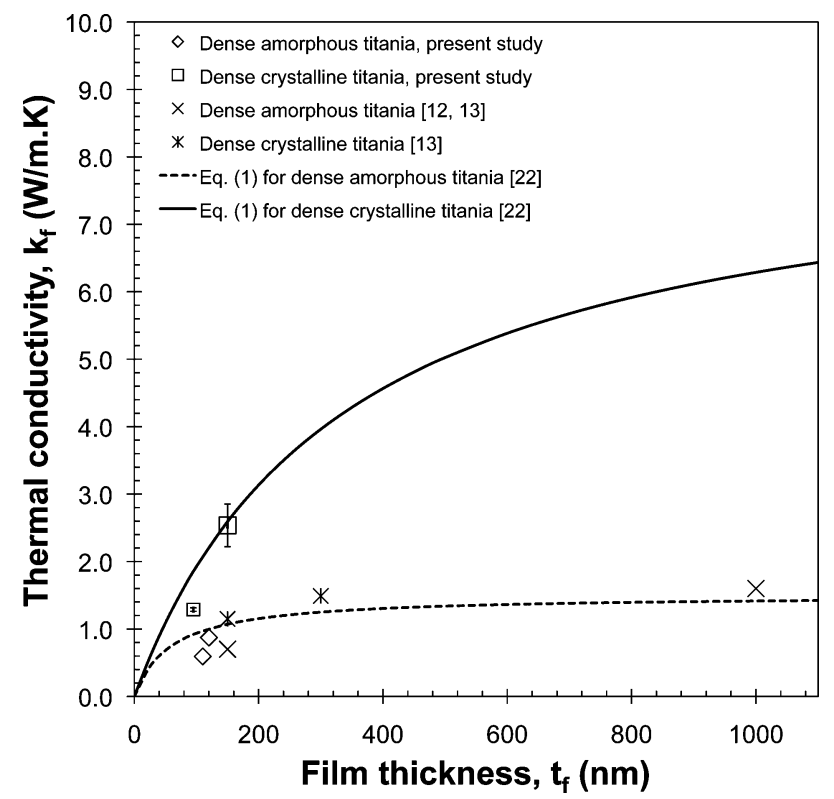

Figure 5. Measured thermal conductivity of dense amorphous $\left(k_{\mathrm{i}}=\right.$ $1.5 \mathrm{~W} / \mathrm{m} \cdot \mathrm{K})$ and crystalline $\left(k_{\mathrm{i}}=8.4 \mathrm{~W} / \mathrm{m} \cdot \mathrm{K}\right) \mathrm{TiO}_{2}$ thin films as a function of film thickness along with previously reported data ${ }^{12,13,22}$ and predictions by eq 1 with $r_{\mathrm{c}}=4.0 \times 10^{-8} \mathrm{~m}^{2} \mathrm{~K} / \mathrm{W}$.

thermal conductivity measurements along with the thickness, porosity, pore size, wall thickness, and crystallinity of each synthesized mesoporous thin films. The reported uncertainty associated with thermal conductivity corresponds to a $95 \%$ confidence interval.

3.1. Sol-Gel Amorphous Mesoporous Titania: Effects of Porosity, Pore Size, and Film Thickness. In this study, the sol-gel amorphous mesoporous titania films exhibited similar porosities of about $30 \pm 2 \%$. Figure 6 shows the thermal conductivity of the mesoporous titania thin films at room temperature as a function of film thickness. Although they were made with two different types of polymers and had different thicknesses, all the amorphous thin films exhibited very similar cross-plane thermal conductivities. The average value was found to be $0.37 \pm 0.05 \mathrm{~W} / \mathrm{m} \cdot \mathrm{K}$ which is $2-3$ times lower than that of dense titania thin films of similar thickness. This can be attributed to a purely geometrical effect resulting from the reduction of cross-sectional area through which heat can diffuse in the amorphous titania matrix. ${ }^{26}$

Moreover, heat in amorphous titania is transported by localized nonpropagating vibrational modes. ${ }^{27,28}$ The spatial extent of those vibrational modes is of the same order of magnitude as the interatomic distance and is estimated to be about $0.6 \mathrm{~nm} .^{29}$ This is at least 10 times smaller than the length scales corresponding to the pore diameter and the wall thickness. The intrinsic thermal conductivity $k_{\mathrm{i}}$ of the $\mathrm{TiO}_{2}$ films is, therefore, independent of those two parameters. In addition, Figure 6 shows that the measured thermal conductivity $k_{\mathrm{f}}$ was also independent of film thickness. As suggested by eq $1, k_{\mathrm{f}}$ depends on the film thickness $t_{\mathrm{f}}$ through the effect of the contact thermal resistance $r_{\mathrm{c}}$. For dense titania thin films, the value of $r_{\mathrm{c}}$ is of the same order of magnitude as the film thermal resistance given by $t_{\mathrm{f}} / k_{\mathrm{i}}$. However, for mesoporous titania thin films, the intrinsic thermal conductivity $k_{\mathrm{i}}$ was dramatically reduced because of the presence of the pores. The interfacial thermal resistance was then about 10 times smaller than the thermal resistance of the mesoporous thin films. Indeed, the interfacial thermal resistance $r_{\mathrm{c}}$ was $4.0 \times 10^{-8} \mathrm{~m}^{2} \cdot \mathrm{K} / \mathrm{W}$ in the present study, while the measured thermal resistance of the 
TABLE 1: Measured Thermal Conductivity and Characteristics of the Synthesized Titania Thin Films

\begin{tabular}{|c|c|c|c|c|c|c|c|c|c|}
\hline $\begin{array}{l}\text { sample } \\
\text { no. }\end{array}$ & crystallinity & process & surfactant & $\begin{array}{l}\text { porosity } f_{\mathrm{v}} \\
\quad( \pm 2 \%)\end{array}$ & $\begin{array}{l}\text { thickness } \\
t_{\mathrm{f}}(\mathrm{nm})\end{array}$ & $\begin{array}{l}\text { pore diameter } \\
\quad d(\mathrm{~nm})\end{array}$ & $\begin{array}{l}\text { wall thickness } \\
t_{\text {wall }}(\mathrm{nm})\end{array}$ & $\begin{array}{l}\text { crystal } \\
\text { size (nm) }\end{array}$ & $\begin{array}{c}\text { conductivity } \\
(\mathrm{W} / \mathrm{m} \cdot \mathrm{K})\end{array}$ \\
\hline 1 & amorphous & sol-gel & - & $0 \%$ & 110 & - & - & - & $0.59 \pm 0.02$ \\
\hline 2 & amorphous & sol-gel & - & $0 \%$ & 120 & - & - & - & $0.87 \pm 0.04$ \\
\hline 3 & polycrystalline & sol-gel & - & $0 \%$ & 95 & - & - & 30 & $1.29 \pm 0.03$ \\
\hline 4 & polycrystalline & sol-gel & - & $0 \%$ & 150 & - & - & 30 & $2.54 \pm 0.32$ \\
\hline 5 & amorphous & sol-gel & P123 & $30 \%$ & 145 & $7-12$ & $3-6$ & - & $0.34 \pm 0.05$ \\
\hline 6 & amorphous & sol-gel & P123 & $30 \%$ & 90 & $7-12$ & $3-6$ & - & $0.38 \pm 0.01$ \\
\hline 7 & amorphous & sol-gel & KLE & $30 \%$ & 155 & $14-19$ & $8-12$ & - & $0.39 \pm 0.01$ \\
\hline 8 & amorphous & sol-gel & KLE & $30 \%$ & 150 & $14-19$ & $8-12$ & - & $0.38 \pm 0.02$ \\
\hline 9 & amorphous & sol-gel & KLE & $30 \%$ & 300 & $14-19$ & $8-12$ & - & $0.48 \pm 0.02$ \\
\hline 10 & amorphous & sol-gel & KLE & $30 \%$ & 260 & $14-19$ & $8-12$ & - & $0.29 \pm 0.02$ \\
\hline 11 & amorphous & sol-gel & KLE & $30 \%$ & 250 & $14-19$ & $8-12$ & - & $0.39 \pm 0.03$ \\
\hline 12 & amorphous & sol-gel & KLE & $30 \%$ & 240 & $14-19$ & $8-12$ & - & $0.32 \pm 0.00$ \\
\hline 13 & amorphous & sol-gel & KLE & $30 \%$ & 240 & $14-19$ & $8-12$ & - & $0.38 \pm 0.00$ \\
\hline 14 & polycrystalline & sol-gel & P123 & $13 \%$ & 60 & $7-30$ & $10-50$ & 9 & $1.21 \pm 0.02$ \\
\hline 15 & polycrystalline & sol-gel & P123 & $13 \%$ & 90 & $7-30$ & $10-50$ & 9 & $1.26 \pm 0.02$ \\
\hline 16 & polycrystalline & sol-gel & KLE & $30 \%$ & 280 & $14-19$ & $8-12$ & $12-13$ & $1.05 \pm 0.06$ \\
\hline 17 & polycrystalline & sol-gel & KLE & $30 \%$ & 260 & $14-19$ & $8-12$ & $12-13$ & $1.02 \pm 0.10$ \\
\hline 18 & polycrystalline & sol-gel & KLE & $30 \%$ & 250 & $14-19$ & $8-12$ & $12-13$ & $1.11 \pm 0.01$ \\
\hline 19 & polycrystalline & sol-gel & KLE & $30 \%$ & 370 & $14-19$ & $8-12$ & $12-13$ & $1.05 \pm 0.01$ \\
\hline 20 & polycrystalline & NC-based ${ }^{a}$ & KLE & $35 \%$ & 95 & $17-25$ & $15-25$ & 9 & $0.44 \pm 0.02$ \\
\hline 21 & polycrystalline & NC-based ${ }^{a}$ & KLE & $35 \%$ & 160 & $17-25$ & $15-25$ & 9 & $0.53 \pm 0.00$ \\
\hline 22 & polycrystalline & NC-based $^{a}$ & KLE & $35 \%$ & 180 & $17-25$ & $15-25$ & 9 & $0.46 \pm 0.00$ \\
\hline
\end{tabular}

${ }^{a}$ Nanocrystal-based.

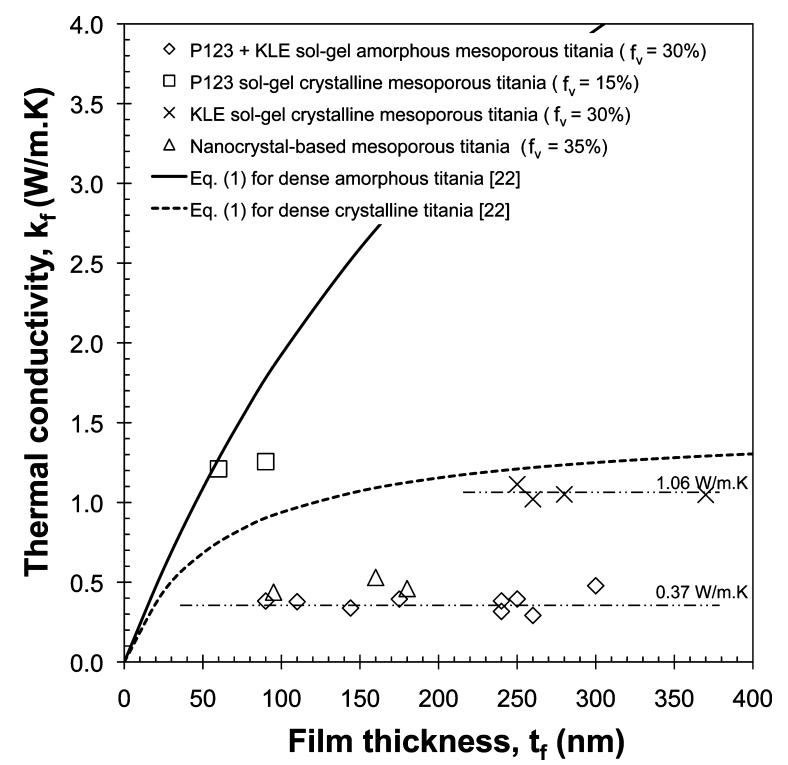

Figure 6. Comparison between the measured cross-plane thermal conductivity of amorphous and crystalline mesoporous $\mathrm{TiO}_{2}$ thin films at room temperature as a function of film thickness. For reference, model predictions by eq 1 for amorphous and crystalline dense films with $r_{\mathrm{c}}=4.0 \times 10^{-8} \mathrm{~m}^{2} \mathrm{~K} / \mathrm{W}$ are also shown.

amorphous mesoporous titania thin films fell between $3.0 \times$ $10^{-7}$ and $4.4 \times 10^{-7} \mathrm{~m}^{2} \cdot \mathrm{K} / \mathrm{W}$. In other words, referring to eq $1, k_{\mathrm{i}} r_{\mathrm{c}} / t_{\mathrm{f}} \approx 1$ for dense films while $k_{\mathrm{i}} r_{\mathrm{c}} / t_{\mathrm{f}} \ll 1$ for mesoporous thin films. Thus, for mesoporous $\mathrm{TiO}_{2}$ films, the measured thermal conductivity corresponded to the mesoporous films' intrinsic thermal conductivity and was independent of thickness.

Finally, while the effect of porosity on thermal conductivity of the amorphous mesoporous $\mathrm{TiO}_{2}$ thin films was not investigated, it is expected to be the same as that already observed for similar polymer-templated mesoporous $\mathrm{SiO}_{2}$ thin films ${ }^{18}$ since heat transfer mechanisms and morphologies are similar. Thermal conductivity should decrease dramatically with increasing porosity.

3.2. Crystalline Mesoporous Titania: Effect of Crystallinity. The effect of polycrystallinity was investigated by comparing the thermal conductivity of the KLE-templated amorphous (samples 7-13) and crystalline (samples 16-19) sol-gel-derived mesoporous titania thin films. Sample porosities and structures were similar, and the effect of crystallinity could thus be isolated. The KLE sol-gel crystalline mesoporous titania thin film had an average thermal conductivity of $1.06 \pm 0.04$ $\mathrm{W} / \mathrm{m} \cdot \mathrm{K}$. This should be compared with $0.37 \pm 0.05 \mathrm{~W} / \mathrm{m} \cdot \mathrm{K}$ for the corresponding amorphous mesoporous films. However, the thermal conductivity of the KLE sol-gel crystalline mesoporous titania thin films remained 3-4 times smaller than that of dense polycrystalline films of same thickness. This can be attributed to (i) the presence of the pores and the reduction of the cross-sectional area through which the heat is transported in the mesoporous titania ${ }^{26}$ and (ii) the small size $(12-13 \mathrm{~nm}$ ) of the crystalline domains in the $\mathrm{TiO}_{2}$ matrix. Indeed, these domains feature very large surface area-to-volume ratios resulting in significant interface thermal resistance to heat transfer. Therefore, the vibrational modes responsible for heat conduction in the crystalline mesoporous films remain very localized and can still not be properly defined as phonons, unlike the case in larger crystals. ${ }^{30}$

The average thermal conductivity of the sol-gel crystalline mesoporous titania thin films made from P123 was found to be $1.24 \pm 0.03 \mathrm{~W} / \mathrm{m} \cdot \mathrm{K}$. It was larger than that of the KLEtemplated films despite having smaller crystalline domains, i.e., $9 \mathrm{~nm}$ instead of $12-13 \mathrm{~nm}$. This can be attributed to the lower porosity of P123-templated films caused by the pore restructuring during crystallization. This establishes that porosity has a stronger influence on thermal conductivity than the size of the crystalline domains. The thermal conductivity of the P123templated crystalline mesoporous titania sol-gel films was similar to that predicted by eq 1 for dense polycrystalline titania thin films of similar thicknesses. This confirms the very low porosity of these films and underlines the uncertainty in the choice of $k_{\mathrm{i}}$ for dense polycrystalline materials, which also depends on the crystal size. ${ }^{23}$

Finally, the thermal conductivity of the nanocrystal-based mesoporous film was measured to be $0.48 \pm 0.05 \mathrm{~W} / \mathrm{m} \cdot \mathrm{K}$. It was slightly larger than that of the KLE and P123 sol-gel amorphous mesoporous films but significantly lower than that of the corresponding sol-gel crystalline mesoporous films. Nanocrystal-based films had a porosity just $5 \%$ larger than that 
of the sol-gel crystalline films, but the nanocrystalline particle's size was smaller ( $9 \mathrm{~nm}$ instead of $13 \mathrm{~nm}$ ) than the crystal size within the matrix of the KLE sol-gel mesoporous films. More importantly, however, is the fact that the nanocrystals were not as well interconnected as the crystalline domains in the sol-gel films. Instead of being a network of crystalline domains, the nanocrystals touch only at points and have significant porosity in between them. Note that the "ultralow" thermal conductivity of nanoparticle packed beds was also investigated by Prasher and co-workers. ${ }^{31,32}$

Taken together, these results indicate a variety of ways to tune thermal conductivity in mesoporous materials. Thermal conductivity of a mesoporous thin film can thus be enhanced by either (i) decreasing porosity, (ii) increasing crystal size, or (iii) enhancing domain connectivity. Porosity and domain connectivity seem to have the greatest influence. The effect of porosity was established in our previous study on mesoporous $\mathrm{SiO}_{2}$ thin films. ${ }^{18}$

\section{Conclusion}

This paper presented preparation, characterization, and crossplane thermal conductivity measurements at room temperature of amorphous and crystalline, dense, sol-gel, and nanocrystalbased mesoporous titania thin films with various thicknesses, pore sizes, porosities, and morphologies. The following conclusions can be drawn:

(1) The average thermal conductivity of amorphous KLEand P123-templated mesoporous sol-gel films with porosity of $30 \%$ was measured as $0.37 \pm 0.05 \mathrm{~W} / \mathrm{m} \cdot \mathrm{K}$ at room temperature.

(2) The thermal conductivity of the amorphous mesoporous titania sol-gel films was found to be independent of film thickness due to the fact that the thermal resistance of the films was much larger than the interface resistance.

(3) Pore diameter and wall thickness did not have any measurable effects on the effective thermal conductivity of the amorphous mesoporous films because of the localized nature of vibrational modes in the $\mathrm{TiO}_{2}$ amorphous matrix.

(4) The effective thermal conductivity of mesoporous sol-gel type $\mathrm{TiO}_{2}$ films increased with crystallinity of the $\mathrm{TiO}_{2}$ matrix. Thermal conductivity of the KLE-templated sol-gel crystalline mesoporous thin films was found to be $1.06 \pm 0.04 \mathrm{~W} / \mathrm{m} \cdot \mathrm{K}$, which is $2-3$ times larger than that of the sol-gel amorphous mesoporous thin films of similar porosity.

(5) Despite the particles' crystallinity, nanocrystal-based films had an average thermal conductivity of $0.48 \pm 0.05$ $\mathrm{W} / \mathrm{m} \cdot \mathrm{K}$, which was significantly lower than that of the sol-gel crystalline mesoporous thin films with slightly lower porosity.

(6) The tuning of both connectivity between nanocrystals and the crystalline domain size can provide control over thermal conductivity in addition to porosity.

Acknowledgment. This material is based upon work supported by the National Science Foundation under Grant CTS 0449429 (L.P.) and CHE-05270 15 (S.H.T) and by Intel/NERC/ UC Discovery Program. T.B. acknowledges the support of a Liebig fellowship by the Fonds der Chemischen Industrie. Portions of this research were carried out at the Stanford Synchrotron Radiation Laboratory, a national user facility operated by Stanford University on behalf of the U.S. Department of Energy, Office of Basic Energy Science.

\section{NOMENCLATURE}

$d$

$d_{\mathrm{k}}$

$d_{\mathrm{k}} \quad$ lattice parameter $[\mathrm{m}]$

$f_{\mathrm{v}} \quad$ porosity

$k_{\mathrm{f}} \quad$ film thermal conductivity $[\mathrm{W} / \mathrm{m} \cdot \mathrm{K}]$

$k_{\mathrm{i}} \quad$ intrinsic thermal conductivity $[\mathrm{W} / \mathrm{m} \cdot \mathrm{K}]$

$r_{\mathrm{c}} \quad$ thermal contact resistance $\left[\mathrm{m}^{2} \cdot \mathrm{K} / \mathrm{W}\right]$

$S \quad$ scattering vector $\left[\mathrm{nm}^{-1}\right]$

$t_{\mathrm{f}} \quad$ film thickness [nm]

$t_{\text {wall }} \quad$ wall thickness [nm]

$\theta \quad$ X-ray diffraction angle [deg]

\section{References and Notes}

(1) Gratzel, M.; O’Regan, B. A low-cost, high-efficiency solar cell based on dye-sensitized colloidal $\mathrm{TiO}_{2}$ films. Nature 1991, 353, 737-740.

(2) Leroux, F.; Dewar, P. J.; Intissar, M.; Ouvrard, G.; Nazarb, L. Study of the formation of mesoporous titania via a template approach and of subsequent Li insertion. J. Mater. Chem. 2002, 12, 3245-3253.

(3) Wang, K.; Morris, M. A.; Holmes, J. D. Preparation of mesoporous titania thin films with remarkably high thermal stability. Chem. Mater. 2005, 17 (6), 1269-1271.

(4) Devi, G. S.; Hyodo, T.; Shimizu, Y.; Egashira, M. Synthesis of mesoporous $\mathrm{TiO}_{2}$-based powders and their gas-sensing properties. Sens. Actuators, B 2002, 87 (1), 122-129.

(5) Hoffmann, M. R.; Martin, S. T.; Choi, W.; Bahnemann, D. W. Environmental applications of semiconductor photocatalysis. Chem. Rev. 1995, 95 (1), 69-96.

(6) Negishi, N.; Iyoda, T.; Hashimoto, K.; Fujishima, A. Preparation of transparent $\mathrm{TiO}_{2}$ thin film photocatalyst and its photocatalytic activity. Chem. Lett. 1995, 24 (9), 841.

(7) Vogel, R.; Meredith, P.; Kartini, I.; Harvey, M.; Riches, J. D.; Bishop, A.; Heckenberg, N.; Trau, M.; Rubinsztein-Dunlop, H. Mesostructured dye-doped titanium dioxide for micro-optoelectronic applications. ChemPhysChem 2003, 4 (6), 595-603.

(8) Fattakhova-Rohlfing, D.; Wark, M.; Brezesinski, T.; Smarsly, B. M.; Rathousky, J. Highly organized mesoporous $\mathrm{TiO}_{2}$ films with controlled crystallinity: A Li-insertion study. Adv. Funct. Mater. 2007, 17, 123-132.

(9) Brezesinski, T.; Wang, J.; Polleux, J.; Dunn, B.; Tolbert, S. H. Templated nanocrystal-based porous $\mathrm{TiO}_{2}$ films for next-generation electrochemical capacitors. J. Am. Chem. Soc. 2009, 131 (5), 1802-1809.

(10) Wang, J.; Polleux, J.; James, L.; Dunn, B. Pseudocapacitive contributions to electrochemical energy storage in $\mathrm{TiO}_{2}$ (anatase) nanoparticles. J. Phys. Chem. C 2007, 111 (6), 14925-14931.

(11) Choi, S. G.; Ha, T.-J.; Yu, B.-G.; Shin, S.; Hee Cho, H.; Park, H.-H. Application of mesoporous $\mathrm{TiO}_{2}$ as a thermal isolation layer for infrared sensors. Thin Solid Films 2007, 516 (2-4), 212-215.

(12) Cahill, D. G.; Hallen, T. H. Thermal conductivity of sputtered and evaporated $\mathrm{SiO}_{2}$ and $\mathrm{TiO}_{2}$ optical coatings. Appl. Phys. Lett. 1994, 65 (3), 309-311.

(13) Mun, J.; Kima, S. W.; Kato, R.; Hatta, I.; Lee, S. H.; Kange, K. H. Measurement of the thermal conductivity of $\mathrm{TiO}_{2}$ thin films by using the thermo-reflectance method. Thermochim. Acta 2007, 455, 55-59.

(14) Lee, S. M.; Cahill, D. G. Thermal conductivity of sputtered oxide films. Phys. Rev. B 1995, 81 (6), 2590-2595.

(15) Kim, D. J.; Kim, D. S.; Cho, S.; Kim, S. W.; Lee, S. H.; Kim, J. C. Measurement of thermal conductivity of $\mathrm{TiO}_{2}$ thin films using $3 \omega$ method. Int. J. Thermophys. 2004, 25 (1), 281-289.

(16) Alberius, P. C. A.; Frindell, K. L.; Hayward, R. C.; Kramer, E. J.; Stucky, G. D.; Chmelka, B. F. General predictive syntheses of cubic, hexagonal, and lamellar silica and titania mesostructured thin films. Chem. Mater. 2002, 14 (8), 3284-3294.

(17) Brezesinski, T.; Groenewolt, M.; Gibaud, A.; Pinna, N.; Antonietti, M.; Smarsly, B. M. Evaporation-induced self-assembly (EISA) at its limit: Ultrathin, crystalline patterns by templating of micellar monolayers. $A d v$. Mater. 2006, 18 (17), 2260-2263.

(18) Coquil, T.; Richman, E. K.; Hutchinson, N.; Tolbert, S. H.; Pilon, L. Thermal conductivity of cubic and hexagonal mesoporous silica thin films. J. Appl. Phys. 2009, 106 (3), 034910.

(19) Cahill, D. G. Thermal conductivity measurement from 30 to 750 K: The $3 \omega$ method. Rev. Sci. Instrum. 1990, 61, 802-808.

(20) Olson, B. W.; Graham, S.; Chen, K. A practical extension of the 3 omega method to multilayer structures. Rev. Sci. Instrum. 2005, 76, 053901.

(21) Jeffreys, H. The stability of a layer of fluid heated from below. Philos. Mag. 1926, 2 (7), 833-844.

(22) Lee, S. M.; Cahill, D. G. Heat transport in thin dielectric films. J. Appl. Phys. 1997, 52 (1), 253-257.

(23) Touloukian, Y. S.; Powell, R. W.; Ho, C. Y.; Klemens, P. G. Thermal Conductivity: Nonmetallic Solids, Vol. 2 of TPRC Data Series; Plenum: New York, 1970. 
(24) Yamane, T.; Nagai, N.; Katayama, S. I.; Todoki, M. Measurement of thermal conductivity of silicon dioxide thin films using a $3 \omega$ method. J. Appl. Phys. 2002, 91 (12), 9772-9776.

(25) Williford, R. E.; Li, X. S.; Addleman, R. S.; Fryxell, G. E.; Baskaran, S.; Birnbaum, J. C.; Coyle, C.; Zemanian, T. S.; Wang, C.; Courtney, A. R. Mechanical stability of templated mesoporous silica thin films. Microporous Mesoporous Mater. 2005, 85, 260-266.

(26) Cahill, D. G.; Stephens, R. B.; Tait, R. H.; Watson, S. K.; Pohl, R. O. Thermal conductivity and lattice vibrations in glasses. In Thermal Conductivity 21, Proceedings of the 21st International Thermal Conductivity Conference, October 15-18, 1989, Lexington, KY; Cremers, C. J., Fine, H. A., Eds.; Springer-Verlag: New York, 1990; pp 3-16.

(27) Shenogin, S.; Bodapati, A.; Keblinski, P.; McGaughey, A. J. H. Predicting the thermal conductivity of inorganic and polymeric glasses: The role of anharmonicity. J. Appl. Phys. 2009, 105, 034906.
(28) Jagannathan, A.; Orbach, R.; Entin-Wohlman, O. Thermal conductivity of amorphous materials above the plateau. Phys. Rev. B 1989, 39 (18), 13465-13477.

(29) Feldman, J. L.; Kluge, M. D.; Allen, P. B.; Wooten, F. Thermal conductivity and localization in glasses: Numerical study of a model of amorphous silicon. Phys. Rev. B 1993, 48 (17), 12589-12602.

(30) Cahill, D. G. Heat transport in dielectric thin films and at solid-solid interfaces. In Microscale Energy Transport; Majumdar, A., Tien, C., Gerner, F., Eds.; Taylor and Francis: Washington, DC, 1997; pp 85-118.

(31) Prasher, R. Ultralow thermal conductivity of a packed bed of crystalline nanoparticles: A theoretical study. Phys. Rev. B 2006, 74 (16), 165413.

(32) Hu, X. J.; Prasher, R.; Lofgreen, K. Ultralow thermal conductivity of nanoparticle packed bed. Appl. Phys. Lett. 2007, 91 (20), 203113.

JP103251T 